\title{
CARACTERÍSTICAS CLÍNICAS, PARACLÍNICAS Y SOCIODEMMOGRAFICAS DE PACIENTES CON CÁNCER DE PRÓSTATA EN EL HOSPITAL UNIVERSITARIO HERNANDO MONCALEANO PERDOMO DE NEIVA
}

Clinical, paraclinical, and sociodemographic prostate cancer patient's at the University Hospital Hernando Perdomo Moncaleano in Neiva

Gustavo Borrero', Claudia González', Gilberto Astaiza". Medico Universidad Surcolombiana, Neiva'.

Profesor Asociado, Departamento de Medicina Social y Preventiva, Facultad de Salud, Universidad Surcolombiana, Neiva².

Correspondencia: e-mail: gastaiza@yahoo.com*

\section{RESUMEN}

Con el objetivo de tener un mejor conocimiento de los pacientes con Cáncer de Próstata en nuestro medio, se realizó un estudio observacional descriptivo, analítico, cualitativo con componente retrospectivo trasversal, en el HUHMP de Neiva durante el 1 de Enero de 2000 y el 31 de Agosto de 2002, que comprendió 45 pacientes con diagnóstico de Cáncer de Próstata confirmado por patología. Se estudiaron variables como: edad, procedencia, seguridad social, antecedente familiar con Cáncer de Próstata, características del tacto rectal, niveles de PSA (antígeno prostático especifico), métodos diagnósticos, presencia de metástasis, características histológicas y tratamiento. Los datos fueron codificados y procesados en el programa Epi Info 2002. No se buscaron relaciones de tipo causal entre las variables, pero se logró establecer relación de covariación entre ellas. Los principales resultados fueron: el 37,8\% de los pacientes provienen de Neiva, el $60 \%$ son del área urbana de cada localidad; los pacientes se encuentran entre los 56 y 89 años, con mas alta frecuencia en mayores de 70 años (67\%), solo 2 pacientes $(4,4 \%)$ presentaron antecedente familiar de Cáncer de Próstata, al momento del diagnóstico los niveles de PSA se encuentran aumentados (100\%) y el tacto rectal muestra próstata grande $(88,9 \%)$ de consistencia pétrea (62\%), el tipo histológico en todos los pacientes es el Adenocarcinoma. Hay evidencia de metástasis ósea $(38,5 \%)$ y pulmonar $(10 \%)$, el tratamiento de elección es la Prostatectomia (47\%) y la Orquidectomía Bilateral (51\%).

\section{PALABRAS CLAVE}

Próstata, Cáncer de próstata, Orquidectomia, Prostatectomia, Adenocarcinoma

\section{ABSTRACT}

A descriptive-observational, analytical and qualitative study with a retrospective crosssectional component was carried out aiming at having a deeper view of próstate cancer patients. It took place at the Hernando Moncaleano Perdomo Hospital in Neiva from January 1st, 2000 to August 31st , 2002.

The sample was made up of 45 patients diagnosed with prostate cancer after pathological tests. The study variable were: age, origin, social security, 
prostate cancer familiar background, rectal tactus caracteristics, level of PSA (Prostatic Specific Antigen), diagnostic methods, metastasis presence, histological characteristics and treatment.

Epi Info 2002 Program was used to code and process data. Cause-type relations were not searched among variabales, but a covariation relation was established among them.

Main results are: $37 \%$ of patients come from Neiva, $60 \%$ of patients live in the urban area, they are aged between 56 and 89, higher frequency was found among patients over 70 years old $(67 \%)$, only two patients $(4,4 \%)$ were found to have prostate cancer familiar background, at the time of the diagnosis test PSA levels were found to be increased $(100 \%)$, and rectal tactus shows a big $(88.9 \%)$ stone consistency prostate gland $(62 \%)$, the histological type in all patients is adenocarcinoma,

There is evidence of bone metastasis $(38,5 \%)$, and lung metastasis $(10 \%)$, the selected treatment was prostatectomy (47\%) and bilateral orchidectomy (51\%).

\section{KEY WORDS}

prostate, prostate cancer, orchidectomy, prostatectomy, adenocarcinoma.

\section{INTRODUCCIÓN}

La próstata tiene como función nutrir el esperma y promover fluidez y el $\mathrm{pH}$ óptimo para la fertilidad y reproducción. Solamente el hombre, el chimpancé y los caninos desarrollan enfermedades en esta glándula. Entre los diferentes mecanismos que participan en las patogénesis de la glándula se destaca la acción hormonal y el envejecimiento ${ }^{1}$.

Actualmente el Cáncer de Próstata $(\mathrm{CaP})$ es la segunda patología con mayor frecuencia dentro de las malignidades del sexo masculino; únicamente superado por el cáncer de piel, siendo además, tras el cáncer de pulmón el segundo en mortalidad ${ }^{2}$. Estudios prospectivos han demostrado la agresividad de este tumor comparando las distintas modalidades terapéuticas que incluyen entre otras la cirugía, radioterapia y terapia expectante, demostrando que esta última es inferior y con una morbilidad mayor, sobre todo, como es lógico esperar, en la población menor de 60 años. El CaP es detectado cada vez más en una fase temprana, por medio de antígeno prostático específico sérico (antígeno especifico prostático). Aunque el PSA es probablemente la mejor prueba de sangre disponible para detectar algún cáncer, todavía ha limitado un poco la especificidad y la sensibilidad ${ }^{3}$.

La mayoría de los pacientes tienen tumores moderadamente bien diferenciados y es probablemente benéfico el tratamiento radical si tiene una buena expectativa de vida de más de 10 años.

Un método nuevo de Prostatectomia radical por laparoscopia parece reducir la morbilidad y la estancia hospitalaria. Las complicaciones de la Prostatectomia han promovido el uso de nuevos tipos de braquiterapia; implantación transperineal de yodo radioactivo o semillas de palladium, han atraído gran publicidad, particularmente en Estados Unidos. Sin embargo, la técnica no está siendo evaluada en un ensayo aleatorio controlado para determinar si es mejor que la espera atenta.

Factores dietarios, genéticos y de estilos de vida contribuyen al desarrollo del cáncer de próstata. Los genes RNASEL y MSR1 heredan susceptibilidad, pueden tener parte en la respuesta a la infección, aumentan la posibilidad de que la infección prostática o la inflamación inicie carcinogénesis prostática ${ }^{4}$.

Epidemiología. Excluyendo el cáncer superficial de piel, el cáncer de próstata es la más común aflicción maligna en hombres Americanos. En 1999, unos 179300 nuevos casos fueron diagnosticados y un estimado de 37000 muertes ocurridas por cáncer de próstata, siendo la segunda causa más común de muerte por cáncer, después del cáncer de pulmón, en hombres Americanos.

Fisiopatología. Factores dietarios, factores del estilo de vida relacionados, y andrógenos han sido largamente reconocidos como contribuyentes al riesgo de cáncer de próstata. Mecanismos nuevamente reconocidos por los cuales carcinógenos medioambientales podían promocionar la progresión de cáncer de próstata y nuevos discernimientos en el camino por el cual receptores andrógenos modulan el fenotipo de células de cáncer de próstata ${ }^{5}$. 
Patología. Microscópicamente la mayoría de los carcinomas de próstata son adenocarcinomas clásicos, condición completa de células epiteliales, con un variado grado de arquitectura glandular ${ }^{5}$.

Aproximadamente en el $70 \%$ de los casos, el carcinoma prostático se origina en la zona periférica de la glándula, clásicamente en una región posterior de esta, que se hace palpable en el tacto rectal. Al corte se observa característicamente que tejido neoplásico es arenoso y firme, pero cuando está incrustado en el parénquima glandular, puede ser sumamente difícil de visualizar y a veces es más fácil palparlo que verlo.

\section{GRADUACIÓN HISTOLÓGICA}

El grado de malignidad, ha sido establecido como un importante marcador de pronóstico en la próstata. El sistema de graduación más ampliamente usado es el de Gleasson, el cual encuentra bajo poder arquitectural, para definir el modelo del tumor. El puntaje de Gleasson da rangos de 2 a 10, y no solo ofrece información significante del pronóstico, sino que permite ser reproducida entre los patólogos.

Elsiguiente estudio buscoidentificar lascaracterísticas sociodemográficas, paraclínicas, clínicas, de diagnóstico y tratamiento de los pacientes con cáncer de próstata en el Hospital Universitario Hernando Moncaleano Perdomo de Neiva.

\section{MATERIALES Y MÉTODOS}

Este es un estudio epidemiológico descriptivo, analítico, cualitativo con componente retrospectivo trasversal. Se llevó a cabo en el Hospital Universitario Hernando Moncaleano Perdomo de Neiva, el cual cuenta con los servicios de: Urgencias, Observación, Hospitalización, UCI y Consulta externa.

La población objeto de estudio fue de 45 historias clínicas, y corresponden a los pacientes con diagnostico de Cáncer de próstata confirmado por patología, que asistieron al Hospital Universitario Hernando Moncaleano Perdomo de Neiva, durante el período comprendido entre el 1 de Enero de 2000 y el 31 de Agosto de 2002. Los datos se obtuvieron del número total de historias clínicas, que cumplieron con ese criterio. Se realizó revisión documental de las historias clínicas de los pacientes. Según las variables, se elaboró un formulario que permitiera obtener la información necesaria para comprender dichas variables; fue diligenciado directamente por los investigadores.

La prueba piloto se realizo aplicando el formulario a 5 historias clínicas escogidas al azar; con el fin de valorar la confiabilidad y validez de este, y para detectar los posibles sesgos de medición con el fin de corregirlos.

Los pacientes del estudio, se diagnosticaron a través de Biopsia transrectal de próstata, Resección transuretral, Prostatectomia, Uretrocistoscopia, Gammagrafía ósea y Radiografía de tórax. Con estos medios, se identifico la patología, tipo histológico y el grado de compromiso general que presentaba el paciente.

Los datos recolectados fueron codificados y procesados en el programa Epi InfoTM. No se buscaron relaciones de tipo causal entre las variables, pero se logró establecer relación de covariación entre ellas.

\section{RESULTADOS}

El área de referencia del hospital pertenece a los departamentos del Huila y Caquetá siendo que la mayor parte de los casos provienen de la ciudad de Neiva y el resto están distribuidos en otros municipios en escasa proporción.

La mayoría de pacientes diagnosticados con $\mathrm{CaP}$ estaban en un rango de edad entre 70 y 80 años de edad, ya pacientes hasta los 60 años de edad y mayores de 86 fueron los menos afectados (Figura 1).

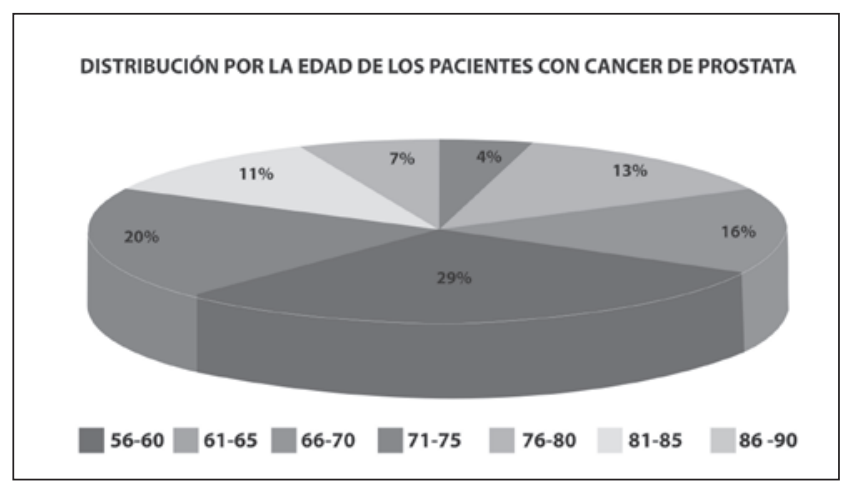

Figura 1. Edad en el momento del diagnostico de los pacientes con cáncer de próstata del Hospital Universitario de Neiva 2000 - 2003. 
Al clasificar estos pacientes por medio de la escala de Gleasson se observo presento con mayor frecuencia el puntaje entre 6 y 7 (Figura 2).

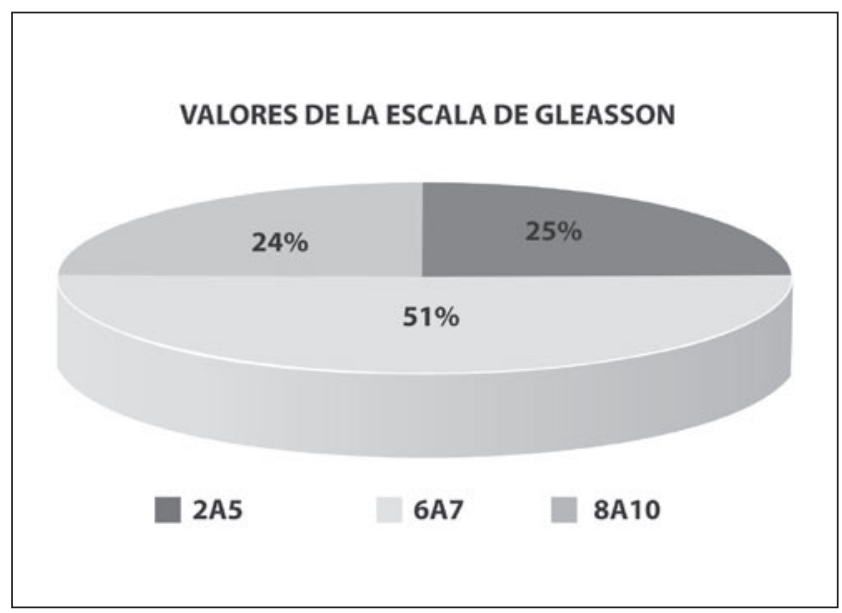

Figura 2. Puntaje en la Escala de Gleasson en los pacientes con cáncer de próstata del Hospital Universitario de Neiva 2000 - 2003.

Inicialmente no se observa re lación entre el desarrollo de cáncer de próstata en los pacientes, y la presencia o ausencia del antecedente familiar de cáncer de próstata (Tabla 1).

Tabla 1. Antecedente de cáncer de Próstata en la familia de los pacientes con cáncer de próstata del Hospital Universitario de Neiva 2000 - 2003.

\begin{tabular}{|c|c|c|}
\hline $\begin{array}{c}\text { CA DE PRÓSTATA } \\
\text { EN LA FAMIIA }\end{array}$ & FRECUENCIA & VALOR (\%) \\
\hline SI & 2 & 4,4 \\
\hline NO & 43 & 95,6 \\
\hline TOTAL & 45 & 100,0 \\
\hline
\end{tabular}

De acuerdo al estadio histológico, al momento de realizado el diagnostico de cáncer de próstata, la mayoría de los pacientes presentaron carcinoma moderadamente diferenciado (58\%) y en muy baja proporción bien diferenciado ( $9 \%$ ); lo que demuestra la detección tardía de la enfermedad, y la imposibilidad de realizar un adecuado control de la misma. Se encontró que el 100\% del cáncer de próstata es del tipo adenocarcinoma (Tabla 2).
Tabla 2. Tipo Histológico en los pacientes con cáncer de próstata del Hospital Universitario de Neiva 2000 - 2003.

\section{TIPO HISTOLÓGICO FRECUENCIA VALOR (\%)}

\begin{tabular}{|c|c|c|}
\hline ADENOCARCINOMA & 45 & 100,0 \\
\hline TOTAL & 45 & 100,0 \\
\hline
\end{tabular}

De forma general los valores séricos de PSA de las muestras fueron elevados: la mayoría de las muestras presento concentraciones superiores $10 \mathrm{ng} / \mathrm{mL}$, mientras que una mínima parte presento concentraciones entre 4 y $10 \mathrm{ng} / \mathrm{mL}$. No hubo concentraciones inferiores a $4 \mathrm{ng} /$ mL. (Tabla 3).

Tabla 3. Niveles de PSA en sangre en los pacientes con cáncer de próstata del Hospital Universitario de Neiva 2000 - 2003

\begin{tabular}{|c|c|c|}
\hline PSA & FRECUENCIA & VALOR $(\%)$ \\
\hline$>10 \mathrm{NG} / \mathrm{ML}$ & 41 & 91,1 \\
\hline $4-10 \mathrm{NG} / \mathrm{ML}$ & 4 & 8,9 \\
\hline TOTAL & 45 & 100,0 \\
\hline
\end{tabular}

Los resultados de la gammagrafía ósea muestran que el compromiso óseo; ya los resultados de la radiografía de tórax muestran compromiso pulmonar y capsular de los pacientes. (Tabla 4 y 5).

Tabla 4. Metástasis en Gammagrafía ósea en los pacientes con cáncer de próstata del Hospital Universitario de Neiva 2000 - 2003.

\begin{tabular}{|c|c|c|}
\hline GAMMA & FRECUENCIA & VALOR (\%) \\
\hline METASTASIS & 10 & 38,5 \\
\hline SI & 16 & 61,5 \\
\hline NO & 26 & 100,0 \\
\hline TOTAL & \\
\hline
\end{tabular}

Tabla 5. Invasión Perineural en los pacientes con cáncer de próstata del Hospital Universitario de Neiva 2000 - 2003.

\begin{tabular}{|c|c|c|}
\hline INVASIÓN PERINEURAL & FRECUENCIA & VALOR (\%) \\
\hline SI & 14 & 31,1 \\
\hline NO & 31 & 68,9 \\
\hline TOTAL & 45 & 100,0 \\
\hline
\end{tabular}

Aproximadamente a la mitad de los pacientes (47\%) se les realizo el procedimiento de prostatectomia radical, y a una fracción de los pacientes no intervenidos (24\%) 
por su estado avanzado de la enfermedad no se le realizo ningún procedimiento quirúrgico y solo se manejo la patología de forma paliativa (Figura 3).

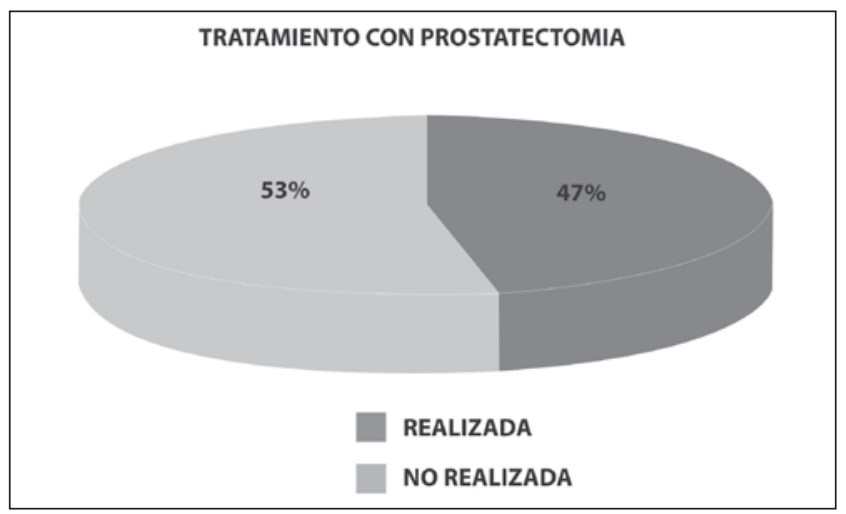

Figura 3. Tratamiento con Prostatectomia en los pacientes con cáncer de próstata del Hospital Universitario de Neiva 2000 - 2003.

\section{DISCUSIÓN}

Una forma importante de la prevención del control del CP es su temprana detección es así como en países con agresivas políticas de tamizaje, como Estados Unidos, se han bajado los índices de cáncer avanzado de un $60 \%$ a mediados de los años 80 , a menos del $30 \%$ en la actualidad ${ }^{5}$.

Parte fundamental del tamizaje es la determinación del PSA (Prostate-specific antigen); como se mencionó en el trabajo. El valor normal de esta prueba, ha venido sufriendo constantes cambios con el tiempo y existe una llamada zona gris del puntaje entre 2 y $10 \mathrm{ng} / \mathrm{dL}$, esto porque no siempre es un indicador directo de la patología ya que el aumento en su concentración puede tener diferentes causas etiológicas PSA, como la hiperplasia prostática, la prostatitis y el adenocarcinoma de próstata, sin existir unidad de criterio para este punto ${ }^{5}$.

Según el National Cancer Institute y la American Cancer Society, si los resultados del PSA son inusuales, su médico puede repetir los exámenes o solicitar una ecografía y otros procedimientos. Estas técnicas de diagnóstico pueden incluir: Ecografía transrectal, Tomografía computarizada, Imágenes por resonancia magnética, Escaneo con radionucleótidos de los huesos, Biopsia de la próstata o de los nódulos linfáticos, o ambos ${ }^{6}$.

En el estudio los valores altos de la prueba del
PSA posiblemente está asociada con una consulta tardía de los pacientes, es decir cuando se presenta la sintomatología relacionada con la patología, lo cual demuestra la falta de pruebas iníciales que permitirían detectar valores del PSA en aumento.

En otras regiones del país se encontró que un $65 \%$ de los casos de CP eran diagnosticados por debajo de $10 \mathrm{ng} / \mathrm{ml}$ y un $25 \%$ por debajo de $4 \mathrm{ng} / \mathrm{ml}$, lo cual permite inferir que se diagnostican casos no indolentes en el curso de la enfermedad ${ }^{7}$.

En estudios previos se evidenció que la edad promedio era menor (61.7 años) con un rango entre los 48 y 70 años, encontrándose el 41.3\% de los afectados por debajo de los 60 años 7. Comparativamente en la región se nota la detección de la patología de edad muy avanzada.

Importante el $\mathrm{CP}$ puede ser afectado o incrementado por otras afecciones, Cajigas y Quiroga analizaron a 127 pacientes con diagnostico de adenocarcinoma de próstata que fueron sometidos a Prostatectomia radical, 23 de ellos, el 29,1\%, presentaban edades superiores a los 70 años, con un rango entre los 70 y los 78 años. De estos, $6(26.08 \%)$ presentaban factores de comorbilidad: $2(8,6 \%)$, sufrían de Hipertensión Arterial, 2 tenían antecedentes de Diabetes Mellitus no insulino dependiente, y 2 más presentaban las dos patologías asociadas ${ }^{8}$.

Entre los factores que contribuyen al cáncer de próstata, se incluye la edad, la raza, la dieta, el peso y la historia familiar. Entre el 5 y el 10 por ciento de los casos de cáncer de próstata, se consideran hereditarios. Sin embargo, la mayoría de los casos son esporádicos (ocurren por casualidad). Aproximadamente el 9 por ciento de todos los cánceres de próstata y el 45 por ciento de los casos en hombres menores de 55 años de edad, pueden atribuirse a un gen susceptible al cáncer que se hereda como característica dominante (de padres a hijos) ${ }^{9}$.

A pesar de los datos que cuestionan su eficacia, la orquidectomía puede ser una forma de tratamiento hormonal. La castración quirúrgica, es un procedimiento ambulatorio que produce una reducción inmediata de las concentraciones de testosterona circulante en un periodo de horas. Algunos datos muestras que puede ser una buena alternativa sin embargo se debe analizar la incidencia de esta sobre la calidad de vida del paciente entre otros factores ${ }^{10}$. 


\section{CONCLUSIONES}

Por medio de un estudio descriptivo retrospectivo de casos, se determino que los pacientes que consultan al Hospital Universitario Hernando Moncaleano Perdomo de Neiva por cáncer de próstata, se encuentran en los estratos socioeconómicos más bajos, cuyas edad oscila entre 56 y 89 años; siendo diagnosticada con mayor frecuencia en edades mayores de 70 años, la mayor parte de los casos provienen de la ciudad de Neiva; casi la totalidad de los pacientes estudiados, presentan sintomatología relacionada con la enfermedad en el momento del diagnostico, concentraciones séricas de PSA elevadas; histológicamente, todos son de tipo adenocarcinoma, por lo cual recomendamos la importancia de plantear medidas de tamizaje, que marquen la diferencia en el diagnóstico, edad y estadio del mismo, que se traduzcan en posibilidades terapéuticas que modifiquen la mortalidad y morbilidad de esta enfermedad, es decir cambiar su historia natural.

\section{REFERENCIAS BIBLIOGRÁFICAS}

1. Weinger WK., Barry MJ. Quality-of-life impact of lower urinary tract symptom severity results from the health professionals follow-up study. Urology. 2002. 59, 245.

2. DuBeau CE. The aging lower urinary tract. Journal of Urology. 2006.175 S11-S15

3. Mifsud A, Choon AT, Fang D, Yong EL. Prostate-specific antigen, testosterone, sexhormone binding globulin and androgen receptor CAG repeat polymorphisms in subfertile and normal men. Molecular Human Reproduction. 2001;7:1007-1013

4. Cybulski C, Wokołorczyk D, Jakubowska A, et al. DNA variation in MSR1, RNASEL and E-cadherin genes and prostate cancer in Poland. Urolology International. 2007. 79; 44- 49.

5. Nelson WG. De Marzo AM.William, Isaac WB; Prostate cancer. The New England Journal of Medicine. 2003. 349; 366-81.

6. Ballentine C, Pearson J, Guess HA, Ross KS. Comparative Efficiency of Prostate-Specific Antigen Screening Strategies for Prostate Cancer Detection. The journal of the American medical association JAMA. 2000; 284:1399-1405.
7. Cajigas JA, Schlesinger R, Hernández J, Rangel J, Quiroga W y Vallejo F. Cáncer de próstata en el hospital militar central. Revista de Urología. Hospital Militar Central.2000.

8. Cajigas JA, Quiroga W. Prostatectomía radical en hombres mayores de 70 años. Revista del Hospital Universitario La Samaritana. 2000.

9. Stanford J, et al. The prostate cancer outcomes study. JAMA. 2000; 283:354-360.

10. Hellerstedt B, Kenneth P. The current state of hormonal therapy for prostate cancer. Cancer Journal Clinicians. 2002; 52:154-179. 